\title{
Preconception and prenatal cystic fibrosis carrier screening of African Americans reveals unanticipated frequencies for specific mutations
}

\author{
Kristin G. Monaghan, $P h D^{1}$, Denise Bluhm ${ }^{1}$, Michelle Phillips ${ }^{2}$, and Gerald L. Feldman, MD, PhD ${ }^{2,3}$
}

\begin{abstract}
Purpose: It is recommended that cystic fibrosis (CF) carrier screening be made available to African Americans who are either pregnant or planning a pregnancy. We analyzed the carrier and mutant allele frequencies for African Americans undergoing CF carrier screening in our laboratories. Methods: Between December 2001 and September 2003, we performed carrier screening for 2189 African Americans, testing for at least the 25 recommended mutations. Results: A total of $33 \mathrm{CF}$ carriers were identified. The most common mutations detected were $\Delta \mathrm{F} 508$, G622D, R117H/7T, and G551D. The G622D allele frequency among African Americans was $0.18 \%$. We did not detect any $3120+1 \mathrm{G} \rightarrow \mathrm{A}$ carriers, although 4 were expected $(P<0.05)$. Conclusions: When considering only the 25 recommended CF mutations, 1 in 75 African Americans screened in our laboratories were carriers (within the expected range, given a $69 \%$ mutation detection rate). The addition of 2 mutations, G622D and Q98R (incidentally identified while screening for ACOG/ACMG mutations), increased the observed carrier frequency to 1 in 66, which is not significantly different from the known African American carrier frequency of 1 in 65 . The frequencies of several specific mutations detected were unanticipated, as was the absence of $3120+1 \mathrm{G} \rightarrow \mathrm{A}$ carriers. Further studies on African American patients with classic CF are needed to examine the incidence of $\mathrm{CF}$ mutations that are not part of the current panel, such as G622D. Genet Med 2004:6(3):141-144.
\end{abstract}

Key Words: cystic fibrosis, African American, carrier screening, G622D, $3120+1 G \rightarrow A$

Cystic fibrosis (CF) is a common autosomal recessive inherited disease, with a carrier rate of 1 in 29 among Caucasians and 1 in 65 among African Americans. The American College of Obstetricians and Gynecologists (ACOG) and the American College of Medical Genetics (ACMG) recommend preconception/prenatal testing for CF carrier status when one or both members of a couple are Caucasian or for any individual with a family history of CF or whose reproductive partner has CF. ${ }^{1,2}$ They also recommend that testing be made available to persons in lower risk groups, including African Americans. The current recommended 25 mutation pan-ethnic panel detects $69 \%$ of mutations in this race, therefore 1 in 94 African Americans is expected to have a positive carrier screen. $\Delta \mathrm{F} 508$ accounts for nearly $50 \%$ of the CF mutations in this race, with $3120+1 \mathrm{G} \rightarrow \mathrm{A}$ accounting for another $12 \%$ of mutations. As the mutation panel may be revised due to changes in the understanding of mutant allele frequencies, the review of allele frequencies in various populations is important. Greater than $1 / 3$ of specimens received in our laboratories for CF testing are

\footnotetext{
${ }^{1}$ Medical Genetics, Henry Ford Hospital, ${ }^{2}$ Detroit Medical Center, University Laboratories, and ${ }^{3}$ Wayne State University, Detroit, Michigan.

Kristin G. Monaghan, PhD, FACMG, Department of Medical Genetics, Henry Ford Hospital, 2799 West Grand Boulevard, CFP 466, Detroit, MI 48202.

Received: November 14, 2003

Accepted: January 30, 2004.
}

DOI: 10.1097/01.GIM.0000127269.42279.83 from African Americans. We discuss our experience with CF carrier screening in African Americans in terms of carrier rates and mutant allele frequencies.

\section{MATERIALS AND METHODS}

\section{Patient population}

Between December 2001 and September 2003, we received a total of 2189 specimens for CF carrier testing among African Americans. This population comprised the second largest racial/ethnic group received in our laboratories, preceded by Caucasians and followed by individuals of Middle Eastern descent. The European admixture for African Americans living in Detroit, Michigan is approximately $20 \% .3,4$

\section{Molecular CF mutation analysis}

DNA was extracted using either the PureGene kit (Gentra) or QIAmp DNA Blood Mini Kit (Quiagen) using conditions specified by the manufacturers. Three methods were used for CF mutation analysis throughout this time period by the two laboratories, and all methods included the 25 mutations recommended by ACOG/ACMG. These methods included heteroduplex analysis, RFLP analysis, CF oligonucleotide ligation assay (OLA) v2.0 and v3.0 (Applied Biosystems), and INNOLiPA CFTR Assay System (Innogenetics). As part of this study, 818 individuals were screened using the CF OLA v2.0 kit to test for $31 \mathrm{CF}$ mutations and a home-brew combination of hetero- 
duplex analysis (exons 4 and 13) and RFLP analysis to test for 3 additional mutations recommended by ACOG/ACMG, but not included in CF OLA v2.0 (I148T, 2184delA, and $3120+1 \mathrm{G} \rightarrow \mathrm{A}$, respectively). During this phase of testing, G622D, Q98R, and variants of unknown clinical significance were identified by heteroduplex analysis as abnormal band shifts and were sequenced in the forward and reverse directions using the dRhodamine dye terminator sequencing kit (Applied Biosystems). Sequencing reactions were run on a 3100 Genetic Analyzer (Applied Bio Systems) and then analyzed using DNA Sequence Analysis Software, Version 3.7 (Applied Bio Systems). In addition, 1274 individuals were tested using the CF OLA v3.0 ASR kit, which includes all 25 recommended mutations (eliminating the need for additional testing using alternative methods) in addition to 7 other CF mutations included in the kit. Detection of all OLA products was performed using an ABI 3100 Genetic Analyzer (capillary sequencer) followed by analysis using GenoTyper software (Applied Biosystems). The INNO-LiPA CFTR Assay System uses PCR derived amplicons, which hybridize to two test strips that contain 64 probes and two control lines. Included on the strips are the mutant and wild-type sequences of 33 immobilized targets covering the 25 mutations recommended in the ACOG/ACMG panel and 8 additional CF mutations. This method was utilized for 97 patients.

Reflex testing for the $3199 \mathrm{del} 6$ and intron 8 polyT locus was performed for carriers of the I148T and R117H mutations, respectively. 3199del6 was tested for by a heteroduplex assay, and the polyT locus was analyzed with either the INNO-LiPA CFTR Assay method or by methods previously described. ${ }^{5}$

\section{RESULTS}

The total number of persons screened, carrier frequency, and specific sequence changes identified using the different methods are summarized in Table 1. Excluding variants of unknown clinical significance, we identified a total of $33 \mathrm{CF}$ carriers among 2189 African Americans undergoing preconception/prenatal screening, an overall observed carrier frequency of 1 in 66 (95\% confidence interval: 1 in 49 to 1 in 100), which is not significantly different from the known African American carrier frequency of 1 in 65. Taking into account only mutations included in the ACOG/ACMG panel, 29 carriers were identified, a frequency of 1 in 75 (95\% confidence interval: 1 in 56 to 1 in 119), which is not significantly different from the expected rate of 1 in 94 (based on a 1 in 65 carrier frequency and $69 \%$ mutation detection rate).

Table 2 shows a summary of the mutant ACOG/ACMG alleles observed in our laboratories between December 2001 and September 2003, compared to mutation frequencies published for $>100$ African Americans with a clinical diagnosis of CF.6,7 As expected, the most common mutation detected was $\Delta \mathrm{F} 508$, which accounted for $61 \%$ of the mutations in our African American population. The next most common mutations detected were G622D (19\%), R117H/7T (12\%), which was increased compared to previous estimates of this mutant allele frequency $(P<0.001)$, and G551D $(6 \%)$. Based on a reported mutant allele frequency of $12 \%$ to $14 \%, 6,7$ we expected to identify the $3120+1 \mathrm{G} \rightarrow \mathrm{A}$ mutation in 4 persons undergoing carrier screening. This mutation was not detected among 4378 African American chromosomes, a result that is statistically significant $(P<0.05)$. Three additional sequence changes, F693L (TTG), Q98R, and P140S(C $\rightarrow$ T), were incidentally detected by heteroduplex analysis while screening for ACOG/ ACMG CF mutations not included in the test kit used during the time of screening. Q98R has been reported to the CF Consortium as a disease causing mutation. F693L (TTG) and $\mathrm{P} 140 \mathrm{~S}(\mathrm{C} \rightarrow \mathrm{T})$ are variants of unknown clinical significance.

\section{DISCUSSION}

It is recommended that $\mathrm{CF}$ carrier screening be made available to African Americans who are either pregnant or considering pregnancy. ${ }^{1,2}$ African Americans comprise a large group of specimens received for carrier screening at our institutions. When considering only the 25 mutation ACOG/ACMG panethnic CF panel, our overall observed carrier frequency of 1 in 75 is within the expected frequency. However, by extending the panel by 2 mutations, we identified an increased number of carriers, 1 in $66 . \Delta \mathrm{F} 508$ was the most common CF mutation identified followed by G622D, R117H/7T, and G551D. R117H has been previously reported at an increased frequency among individuals undergoing carrier screening compared to those with a diagnosis of cystic fibrosis. ${ }^{8}$ An unexpected result was the lack of $3120+1 \mathrm{G} \rightarrow \mathrm{A}$ carriers, although 4 were expected given that this mutation accounts for $\approx 12 \%$ of the CF muta-

Table 1

Summary of carrier screening results using various methods employed between December 2001 and September 2003

\begin{tabular}{|c|c|c|c|}
\hline & $\begin{array}{l}\text { OLA v2.0, heteroduplex analysis (exons } 4 \text { and } 13) \text { and } \\
\text { RFLP analysis }(3120+1 \mathrm{G} \rightarrow \mathrm{A})\end{array}$ & OLA v3.0 & INNO-LiPA \\
\hline Total screened & 818 & 1274 & 97 \\
\hline No. of carriers identified & 16 & 14 & 3 \\
\hline Observed carrier frequency & $1 / 51$ & $1 / 81$ & \\
\hline Mutations identified & $\begin{array}{c}\Delta \mathrm{F} 508 \text { (6), G622D (3), R117H/7T (3), I148T (3199del6 } \\
\text { negative), Q98R, 1898+1G } \rightarrow \text { A, and G551D }{ }^{a}\end{array}$ & $\Delta$ F508 (14), R117H/7T, R553X, and G551D & \\
\hline
\end{tabular}

${ }^{a}$ In addition, 2 persons were positive for F693L (TTG) and 1 was positive for $\mathrm{P} 140 \mathrm{~S}$ (C $\rightarrow \mathrm{T}$ at 550 ); both are variants of unknown clinical significance. 
Table 2

Summary of ACOG/ACMG CF mutations identified among 2189 African Americans (4378 chromosomes) undergoing carrier screening

\begin{tabular}{|c|c|c|c|}
\hline Mutation & $\begin{array}{l}\text { Carriers } \\
\text { identified }\end{array}$ & $\begin{array}{l}\text { Mutation frequency } \\
(\%) \text { (this study) }\end{array}$ & $\begin{array}{l}\text { Published mutation } \\
\text { frequency }(\%)^{6,7}\end{array}$ \\
\hline$\Delta \mathrm{F} 508$ & $20 / 33$ & 61 & $29^{a}-48$ \\
\hline $\mathrm{R} 117 \mathrm{H} / 7 \mathrm{~T}$ & $4 / 33$ & 12 & 1 \\
\hline G551D & $2 / 33$ & 6 & 1 \\
\hline I148T (3199del6 negative) & $1 / 33$ & 3 & 0 \\
\hline $1898+1 \mathrm{G} \rightarrow \mathrm{A}$ & $1 / 33$ & 3 & 1 \\
\hline R553X & $1 / 33$ & 3 & 0.5 \\
\hline $3120+1 \mathrm{G} \rightarrow \mathrm{A}$ & 0 & 0 & $12-14$ \\
\hline
\end{tabular}

${ }^{a}$ The lower frequency of $\Delta \mathrm{F} 508$ reported by Heim et al. was most likely due to ascertainment bias.

tions in this race. It is not known why the $3120+1 \mathrm{G} \rightarrow \mathrm{A} \mathrm{mu}$ tation was lacking among our African American population undergoing CF carrier screening, although this may be due to random error due to a relatively small sample size. Among African American CF patients tested in our laboratories, this mutation accounts for $\approx 17 \%$ of the CF mutations identified. Therefore, this mutation is present at the expected frequency among African Americans in Detroit who are affected with cystic fibrosis. We do not recommend that this mutation be removed from the ACOG/ACMG CF mutation panel.

Two mutations, G622D and Q98R, and two variants of unknown clinical significance, F693L (TTG) and P140S $(\mathrm{C} \rightarrow \mathrm{T})$, which are not part of the recommended CF screening panel, were incidentally detected while using heteroduplex analysis to screen for ACOG/ACMG recommended mutations (Table 2). Heteroduplex analysis was retired from our laboratory CF protocol in 2002 with the introduction of CF OLA v3.0. Heteroduplex analysis identifies sequence changes based on differences in electrophoretic mobility of PCR amplicons containing mismatches, small insertions, and small deletions compared to a normal homozygous sequence. PCR amplicons producing abnormal band shifts are then sequenced to identify the particular sequence change present.

One of the advantages of heteroduplex analysis and direct DNA sequencing is that these methods will theoretically detect any sequence change present in a PCR amplicon. A disadvantage is the finding of variants of unknown clinical significance, especially when a pregnancy is involved. The finding of a disease-causing CF mutation in a fetus that also has a variant of unknown clinical significance creates a difficult genetic counseling situation. This is the case with F693L (TTG), which is currently classified as a CF mutation, reported on one Hispanic CF chromosome in a patient who also had the $5 \mathrm{~T}$ allele (whether the two mutations were in-trans versus in-cis was not reported). ${ }^{9}$ However, a different base substitution in the same codon resulting in the same amino acid substitution, F693L (CTT), originally reported as a CF mutation, ${ }^{10}$ was also reported as a polymorphism. ${ }^{9}$ This mutation was later shown by functional studies to not affect the CFTR chloride channel ac- tivity or protein maturation. ${ }^{11}$ Therefore, the significance of finding F693 (TTG) in our African American population is not clear. P140S $(\mathrm{C} \rightarrow \mathrm{T})$ has been reported in a patient with disseminated bronchiectasis, although it is not known if this mutation is associated with classic CF. ${ }^{9}$ Q98R has been reported previously as a CF mutation, ${ }^{12}$ although we are not aware of any reports of this mutation in African Americans.

Functional studies have demonstrated abnormal chloride channel activities for G622D. ${ }^{11}$ However, this mutation has been only reported in one patient with oligospermia and no other identifiable CF mutation on the opposite chromosome, ${ }^{9}$ so it is not known if this mutation is associated with classic CF. We have identified this mutation in 5 individuals over the past 2 1/2 years. One case was an African American with features suggestive of CF (borderline sweat chloride test and asthma) who did not have an identifiable CF mutation on the other chromosome, although complete CFTR gene sequencing was not performed on this patient. Three healthy African Americans and one Asian undergoing carrier screening were identified as heterozygotes for this mutation. A total of 4088 individuals (pan-ethnic), including 818 African Americans, have been screened for G622D. Excluding the earlier-mentioned case with a possible diagnosis of $\mathrm{CF}$, the mutant allele frequency among African Americans is $0.18 \%$ and among all races/ethnic groups screened is $0.05 \%$.

Several studies have examined CF mutations in African American (142 cases) $)^{6,7}$ and African ( 4 cases $)^{13}$ patients with a clinical diagnosis of CF. In addition to $\Delta \mathrm{F} 508$ and $3120+1 \mathrm{G} \rightarrow \mathrm{A}$, which are both included in the current mutation panel, other mutations outside of the ACOG/ACMG panel have been reported in African Americans $(405+3 \mathrm{~A} \rightarrow \mathrm{C}$, 444delA, $\Delta$ F311, G480C, A559T, 2307insA, 196del54, G1249E, S1255X and D1270N $\left.\mathrm{N}^{6,7,13,14,15}\right)$. It is interesting that while heteroduplex analysis was used in the laboratory, we did not identify 444delA (exon 4) or 2307insA (exon 13) among our African Americans undergoing CF testing, although this method would likely detect any insertion or deletion mutation present. These mutations are of potential interest to laboratories performing CF carrier screening. We are unaware of any largescale studies of CF mutations in the unaffected African American population.

The current ACOG/ACMG-recommended CF mutation panel is based on alleles with a frequency of $\geq 0.1 \%$ in the general population. However, as expected, due to differences in allele frequencies among persons of different ethnic and racial groups, when panels are examined in a particular population, mutation detection rates are variable. One study showed that extending the current CF mutation panel by 6 common African American mutations would increase the CF mutation detection rate by approximately $1.2 \% .{ }^{14}$ Increasing the number of recommended mutations in the current panel rather than developing ethnic- or racial-specific panels may be beneficial because it is not practical for clinical diagnostic laboratories to assess the specific ethnic/racial background of each specimen it receives. However, laboratories, which receive a large proportion of specimens from individuals with a lower 
risk of being CF carriers, may be especially interested in using a CF panel that is tailored to their patient population, to provide a better detection rate.

The current CF mutation panel is likely to be modified as additional racial and ethnic specific information regarding CF mutations becomes available. As we have learned from I148T,16,17 it is of limited benefit to screen for sequence changes that do not result in a classic CF phenotype. Screening panel mutations should be based on mutations in affected individuals, not necessarily those incidentally identified in the general population. Further studies are needed on the incidence of CF mutations that are not part of the current panel, including G622D, to give an estimate of the true frequency of these alleles in African Americans and the general population in the United States. This testing should include individuals with classic CF.

\section{References}

1. Grody WW, Cutting GR, Klinger KW, Richards CS, Watson MS, Desnick RJ. Laboratory standards and guidelines for population-based cystic fibrosis carrier screening. Genet Med 2001;3:149-154.

2. American College of Obstetrics and Gynecology, and American College of Medical Genetics. Preconception and prenatal carrier screening for Cystic Fibrosis. Clinical and laboratory guidelines. Washington DC: American College of Obstetrics and Gynecology, 2001.

3. Reed TE. Caucasian genes in American Negroes. Science 1969;165:762-768.

4. Parra EJ, Marcini A, Akey J, Martinson J, Batzer MA, Cooper R et al. Estimating African American admixture proportions by use of population-specific alleles. Am J Hum Genet 1998;63:1839-1851.
5. Chillon M, Casals T, Mercier B, Bassas L, Lissens W, Silber S et al. Mutations in the cystic fibrosis gene in patients with congenital absence of the vas deferens. $N$ Engl J Med 1995;332:1475-1480.

6. Macek M, Mackova A, Hamosh A, Hilman BC, Selden RF, Lucotte G et al. Identification of common cystic fibrosis mutations in African-Americans with cystic fibrosis increases detection rate to 75\%. Am J Hum Genet 1997;60:1122-1127.

7. Heim RA, Sugarman EA, Allitto BA. Improved detection of cystic fibrosis mutations in the heterogeneous US population using an expanded, pan-ethnic mutation panel. Genet Med 2002;3:168-176.

8. Strom CM, Huang D, Buller A, Redman J, Crossley B, Anderson B et al. Cystic fibrosis screening using the College panel: Platform comparison and lessons learned from the first 20,000 samples. Genet Med 2002;4:289-296.

9. Hospital for Sick Children, Toronto. Cystic Fibrosis Mutation Database. Available at: http://www.genet.sickkids.on.ca/cftr/

10. Audrezet MP, Novelli G, Mercier B, Sangiuolo F, Maceratesi P, Ferec C et al. Identification of three novel cystic fibrosis mutations in a sample of Italian cystic fibrosis patients. Hum Hered 1993;43:295-300.

11. Vankeerberghen A, Wei L, Jaspers M, Cassiman J-J, Nilius B, Cuppens H. Characterization of 19 disease-associated missense mutations in the regulatory domain of the cystic fibrosis transmembrane conductance regulator. Hum Molec Gen 1998;7: 1761-1769.

12. Romey MC, Desgeorges M, Ray P, Godard P, Demaille J, Claustres M. Novel missense mutation in the first transmembrane segment of the CFTR gene (Q98R) identified in a male adult. Hum Mutat 1995;6:190-191.

13. Carles S, Desgeorges M, Goldman A, Thiart R, Guittard C, Kitazos CA, deRavel TJL, Westwood ATR, Claustres M, Ramsay M. First report of CFTR mutations in black cystic fibrosis patients of southern African origin. J Med Genet 1996;33:802-804.

14. Bobadilla JL, Macek M, Fine JP, Farrell M. Cystic fibrosis: A worldwide analysis of CFTR mutations-correlation with incidence data and application to screening. Hum Mutat 2002;19:575-606.

15. Padoa C, Goldman A, Jenkins T, Ramsay M. Cystic fibrosis carrier frequencies in populations of African origin. J Med Genet 1999;36:41-44.

16. Rohlfs EM, Zhou Z, Sugarmen EA, et al. The I148T CFTR allele occurs on multiple haplotypes: a complex allele is associated with cystic fibrosis. Genet Med 2002;4:319323.

17. Watson MS, Desnick RJ, Grody WW et al. Cystic fibrosis carrier screening: issues in implementation. Genet Med 2002;4:1. 